d'un bouc de dix mois qui, pour avoir été quatre semaines à côté d'une vache à tuberculose ouverte, tomba malade. La chèvre ne possède pas une tuberculose propre (spécifique), mais tombe malade par l'absorption du bacille du type bovin. L'infection tuberculeuse de chèvre à chèvre (par suite de la vie en commun dans la même étable) paraît être, d'après mes observations, un cas rare. Et dans les grandes étables de chèvres, l'infection s'étend beaucoup moins que dans les étables de bovins. C'est que les vaches se trouvent tête contre tête, surtout pendant le manger, et le danger d'infection est très grand avec des animaux toussant fortement. De plus, les vaches tuberculeuses sont longtemps conservées, surtout si elles donnent beaucoup de lait. Et la tuberculose ouverte du bœuf paraît être plus forte que celle de la chèvre.

La chèvre ne peut pas infecter une écurie aussi facilement que la vache, vu que, en moyenne, sa vie est beaucoup plus courte que eelle de la vache. Des recherches serrées de la tuberculose caprine seraient nécessaires au point de vue pratique, clinique, pathologique, bactériologique et épidémique.

Les études scientifiques devraient se porter sur une maladie aussi importante de la chèvre, en remerciement aux éleveurs de chèyres qui s'efforcent d'augmenter la production laitière.

\title{
Bibliographie
}

[1] R. Springreld, Zeitschrift für Ziegenzucht, P. 363, 1912.

[2] Eichноrn, Diagnostiche Tuberkulinimpfungen bei Ziegen (Tuberculination de la chèvre). Sächsische Veterinärberichte, p. 163, 1892.

[3] K. Hertha, Beitrag zur Kenntnis der Ziegentuberkulose, Arbeiten aus dem hygien. Institut der Tierarztl. Hochschule, XVI, Berlin.

[4] Fronner-Zwick, Lehrbuch der speziellen Pathologie und Terapie der Haustiere, T. II, $2^{\mathrm{e}}$ partie, p. 204, 1920.

[5] Schleges, Tuberkulose bei Ziegen. Berl. Tierärztl. Wochenschrift, p. $695,1913$.

[6] Zeitschrift für Ziegenzucht, no 6, 1905.

\section{LE PICA DE LA CHĖVRE (aLlotriophagie)}

\author{
Par le Dr G. FLÜCKIGER \\ Expert de l'Office vétérinaire fédéral, Berne.
}

De même que chez les bovins, le pica peut être observé occasionnellement dans certaines contrées sur les chèvres maintenues en stabulation permanente et nourries d'une manière irrationnelle. L'affection se manifeste par des troubles dans l'assimilation avec une tendance maladive des animaux à lécher les objets les plus divers, à les ronger et, si possible, à les avaler. Des troubles chroniques de la digestion et de la nutrition se produisent simultanément avec 
amaigrissement successif, auxquels s'ajoutent dans beaucoup de cas des phénomènes nerveux.

Les causes principales du pica résident comme nous l'avons déjà dit dans une stabulation contimue et une alimentation défectueuse. La preuve en est fournie par le fait que dans nos régions montagneuses et dans les contrées à pâturages suffisants, cette affection y est totalement inconnue. En outre, les symptômes du piea s'observent beaucoup moins souvent pendant l'affouragement au vert qu'en hiver. L'insuffisance de sels minéraux dans les aliments semble jouer le rôle essentiel dans l'éclosion de la maladie. Les animaux cherchent alors à récupérer les sels manquants de n'importe quelle manière, ce qui expliquerait leur façon de se comporter vis-à-vis de certains objets hétéroclites. Les premiers symptômes se manifestent le plus souvent par une diminution d'appétit, par des troubles dans la rumination et dans la digestion ainsi que par une régression dans la production du lait. Les évacuations intestinales se font irrégulièrement, les excréments se présentent souvent sous forme de grosses boules recouvertes de mucosités, ils sont parfois mous et fétides. En même temps, on observe souvent des marques de frayeur et une grande impressionnabilité ; le regard devient inquiet; les animaux montrent une grande sensibilité de certaines parties du corps, spécialement de la région du dos.

La perversion du goût et le pica ne s'établissent que peu à peu, les animaux montrant alternativement le désir ou le dégoût de certaines sortes d'aliments. Dans beaucoup de cas, le foin est délaissé pour la paille ou d'autres litières. Bien des chèvres lèchent et rongent continuellement les parois, les crèches, les seaux, les colliers, le poil d'animaux voisins, etc. Par la suite, on observe l'amaigrissement, le hérissement du poil, l'atrophie des muscles, l'anémie et avec le temps l'animal tombe dans un état cachectique profond.

Lorsque la maladie est avancée, on observe quelquefois une élévation de la température avec augmentation de la fréquence du pouls; par contre, peu de troubles respiratoires. Chez quelques animaux, il peut y avoir de la paraplégie, du grincement de dents et du ptyalisme prononeé. Hess a constaté dans certains eas que le lait des chèvres atteintes de pica subit des transformations telles que : goût désagréable avee tendance à la coagulation, crème plus épaisse et de couleur douteuse. D'après une communication d'un confrère, les chèvres portantes atteintes de pica sont sujettes à l'avortement. L'ingestion de corps étrangers a souvent pour conséquence une gastrite traumatique. La marche de la maladie a toujours un caractère chronique.

Le régime alimentaire peut amener une amélioration temporaire ou permanente. Avec une alimentation défectueuse persistante, LB LAIT, I926 
l'affection dure en règle générale plusieurs semaines ou même des mois pour se terminęr, après un état eachectique bien prononcé, par la mort. Si, par contre, les animaux malades sont placés en temps voulu dans des conditions favorables, avec un changement approprié dans la nourriture, la maladie disparaît le plus souvent lorsque l'état cachectique n'est pas trop avancé. Dans ce dernier cas et en particulier si la sous-alimentation est trop prononcée, un traitement a peu de chance de réussite. Lorsqu'il en est ainsi, l'abatage à bref délai est la mesure la plus rationnelle.

Dans les premières phases par contre, l'affection peut être combattue facilement par un changement d'alimentation et éventuellement par un changement d'habitat. L'affouragement en bon foin provenant d'une autre région, même distribué en quantité relativement faible, produit souvent un résultat surprenant.

Le régime de pâturage est le meilleur traitement de la maladie chez les animaux en stabulation; il faudrait si possible, les mettre immédiatement au pâturage. Lorsque le changement de régime ou la mise au pâturage ne sont pas possibles, la maladie peut être combattue par l'adjonetion d'une nourriture fortifiante et de sels inorganiques. Comme fortifiants, on donnera du foin de trèfle, du bon foin de prairie et d'esparcette, du lupin séché, un mélange de graine de lin ou de tourteau de lin, de la mélasse, des graines et de la farine de céréales, des racines fourragères et, si possible, du lait pur. Les sels minéraux recommandés sont : le sel de cuisine, la magnésie, les earbonates ou biearbonates de soude en dose de 10 à $20 \mathrm{gr}$. par jour, répartis sur deux à trois rations alimentaires. Dans les régions pauvres en sels calcaires, les sels de chaux (phosphate de chaux) rendent souvent de bons services. Dans d'autres cas, l'huile de foie de morue, la farine artificielle ou l'adjonction d'acide chlorhydrique dans l'eau de boisson se sont montrés efficaces.

Je ne puis me prononcer sur le traitement au ehlorhydrate d'apomorphine recommandé contre le pica de la chèvre par divers auteurs. Par contre, dans les cas de pica qui me sont connus, l'affection a pu, dans la règle, être maîtrisée par les mesures et traitements indiqués ci-dessus.

On admet généralement que les jeunes animaux sont plus prédisposés à l'affection que les adultes. A ma connaissance, la forme enzootique du pica de la chèvre signalée à diverses reprises dans la littérature n'a jamais été constatée en Suisse. 